\title{
CONSIDERATIONS ON RELATIONSHIP BETWEEN EACH SENSITIVITY TERM AND RESULTS OF TOPOLOGY OPTIMIZATION IN UNSTEADY OSCILLATION PROBLEM FOR 3D CANTILEVER BEAM
}

\author{
MASAYUKI KISHIDA $^{1}$ AND TAKAHIKO KURAHASHI ${ }^{1}$ \\ ${ }^{1}$ Nagaoka University of Technology \\ 1603-1, Kamitomioka, Nagaoka, Niigata 940-2188, Japan \\ E-mail s173029@stn.nagaokaut.ac.jp
}

Key words: Topology optimization, Density method, Self-adjoint, Sensitivity terms

\begin{abstract}
In this study, we carried out the topology optimization based on the density method in the unsteady oscillation problems. This paper considers two items, the proposal of new performance function and the relationship between each sensitivity term and results of topology optimization. In other studies, the adjoint analysis must be performed for the propose of minimizing strain energy. Optimization problems are classified into the problem of maximizing work in the negative and the problem of minimizing work in the positive, and self-adjoint relationships are derived. When using this method for cantilever beam model, there is a problem that numerous high-density elements is distributed at the load point, i.e., the tip. By classifying the sensitivity, it is found that the mass term has a great influence on the problem. In our study, some results are shown by changing the penalization parameter in the SIMP method that determines the mass coefficient, damping coefficient and elastic coefficient.
\end{abstract}

\section{INTRODUCTION}

Topology optimization is especially useful in improving the performance and reducing the weight of products [1]. In other researches, the performance function for the unsteady oscillation problems is set to the natural frequency or the square of strain energy[2]. Thus, it is to find the optimal structure without adjoint analysis in topology optimization for unsteady oscillation problem. In this paper, a new performance function is proposed, and self-adjoint relationships are derived by classifying optimization problem into maximization and minimization problem. More details will be given in the next chapter. When the above method is applied to the optimization problem, a lot of density elements are distributed at the load point in the cantilever beam model. The reason is that when the sensitivity is classified into three terms, i.e., terms including mass matrix, terms including damping matrix and terms including stiffness matrix, the sensitivity of the terms including stiffness matrix is high at the load points (See Figure 1). From Figure 1, in order to clarify the relationship between each sensitivity term and the results of topology optimization, the penalization parameters of the Solid Isotropic Material with Penalization (SIMP) method, which is one of the density method, is changed and investigated. Chapters 2 and 3 describe the formulation and the flowchart for topology optimization in the unsteady oscillation problems, and Chapter 4 describes an example of numerical experiments. 


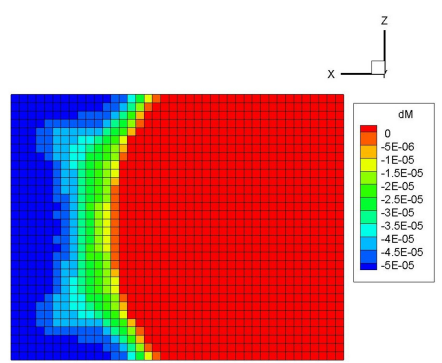

(a)Only terms including mass matrix.

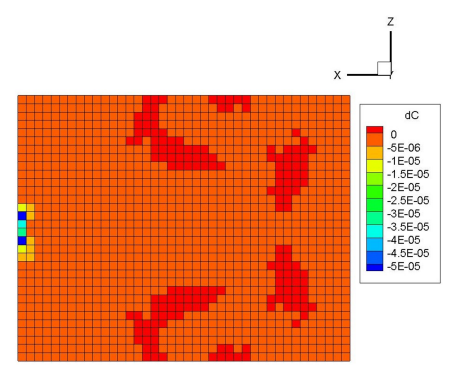

(b)Only terms including damping matrix.

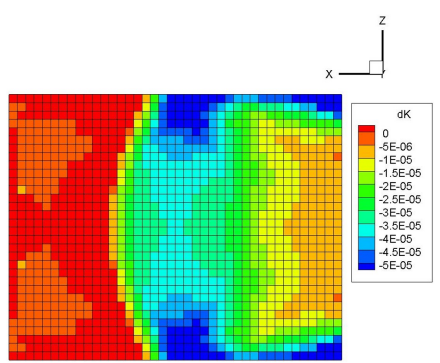

(c)Only terms including stiffness matrix.

Figure 1: Sensitivity distributions of each term at the first iteration.

\section{FORMULATION FOR TOPOLOGY OPTIMIZATION}

This chapter describes the formulation for topology optimization in the unsteady oscillation problems. The aim of this optimization analysis is the minimizing of the strain energy that satisfies the target volume. The strain energy is usually zero when summed over the entire time. Thus, the strain energy can be classified into the work in the positive and the work in the negative. The performance function is set in which the work in the positive is a minimization problem and the work in the negative is a

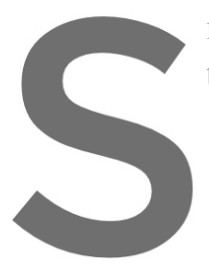
maximization problem the density constraint.
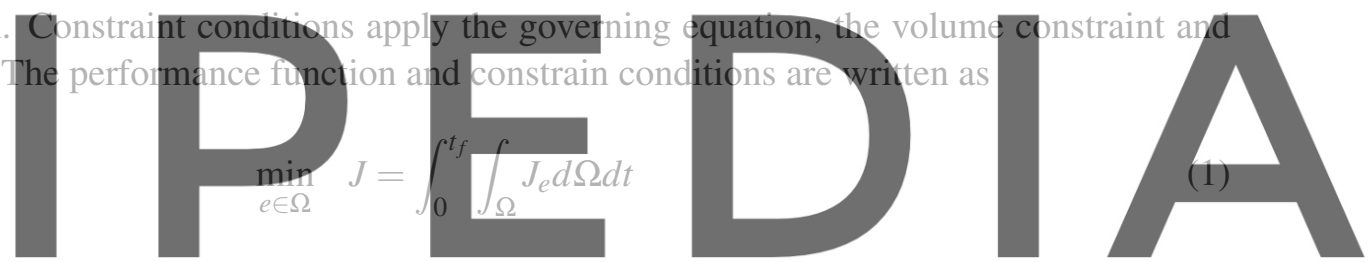

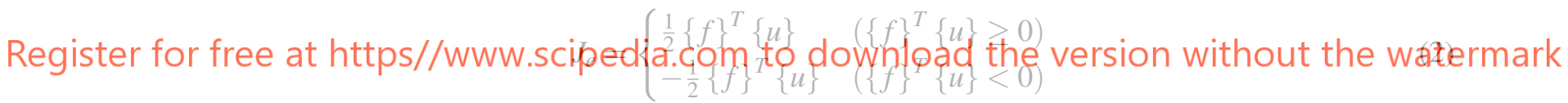

$$
\begin{gathered}
\text { subject to } \int_{0}^{t_{f}} \int_{\Omega}\left[M\left(\rho_{e}\right)\right]\{\ddot{u}\}+\left[C\left(\rho_{e}\right)\right]\{\dot{u}\}+\left[K\left(\rho_{e}\right)\right]\{u\} d \Omega d t=\int_{0}^{t_{f}} \int_{\Omega}\{f\} d \Omega d t \\
V=\int_{\Omega} \frac{\rho_{e} v_{e}}{V_{\text {total }}} d \Omega-\overline{\rho_{0}} \leq 0 \\
0 \leq \rho_{e} \leq 1
\end{gathered}
$$

where $[M],[C],[K],\{\ddot{u}\},\{\dot{u}\}$ and $\{u\}$ are, respectively, the mass matrix, damping matrix, stiffness matrix, acceleration vector, velocity vector and displacement vector. And, $e, t_{f}, \rho, v, V_{\text {total }}$ and $\overline{\rho_{0}}$ are, respectively, the element number, final time, non-dimensional density, an element volume, sum of element volumes in the design domain $\Omega$ and average initial density in the design domain $\Omega .[M],[C]$ and $[K]$ are assumed to be a function of density $\rho_{e}$ in the element $e$. Here, the final time $t_{f}$ is set as enough time for the displacement to be infinitely close to zero. The Lagrange multiplier method is used to determine the density gradient, as shown in Equation (6). In order to obtain the displacement gradient, it is divied into the case of the minimization problem and the case of minimization problem, that is, 
the case of work in the positive and the case of work in the negative. For the work in the positive, the displacement gradient can be calculated as in Equation (7), and for the work in the negative, the displacement gradient can be calculated as Equation (8). From Equations (7) and (8), the self-adjoints are $\{\lambda\}=-\{u\}$ and $\{\lambda\}=\{u\}$, respectively. It is not necessary to perform the adjoint analysis by using the self-adjoint relationships, as shown in Equation (9).

$$
\begin{aligned}
L & =\int_{0}^{t_{f}} \int_{\Omega} J_{e}+\frac{1}{2}\{\lambda\}^{T}\left(\left[M\left(\rho_{e}\right)\right]\{\ddot{u}\}+\left[C\left(\rho_{e}\right)\right]\{\dot{u}\}+\left[K\left(\rho_{e}\right)\right]\{u\}-\{f\}\right) d \Omega d t \\
& =\int_{0}^{t_{f}} \int_{\Omega} L_{e} d \Omega d t
\end{aligned}
$$

$$
\frac{\partial L_{e_{p}}}{\partial u}=\sum_{+} \frac{1}{2} \int \frac{\partial\{u\}^{T}}{\partial u}\left(\{f\}+\left[M\left(\rho_{e}\right)\right]\{\ddot{\lambda}\}-\left[C\left(\rho_{e}\right)\right]\{\dot{\lambda}\}+\left[K\left(\rho_{e}\right)\right]\{\lambda\}\right) d t=0
$$

$$
\frac{\partial L_{e_{n}}}{\partial u}=\sum \frac{1}{2} \int \frac{\partial\{u\}^{T}}{\partial u}\left(-\{f\}+\left[M\left(\rho_{e}\right)\right]\{\ddot{\lambda}\}-\left[C\left(\rho_{e}\right)\right]\{\dot{\lambda}\}+\left[K\left(\rho_{e}\right)\right]\{\lambda\}\right) d t=0
$$

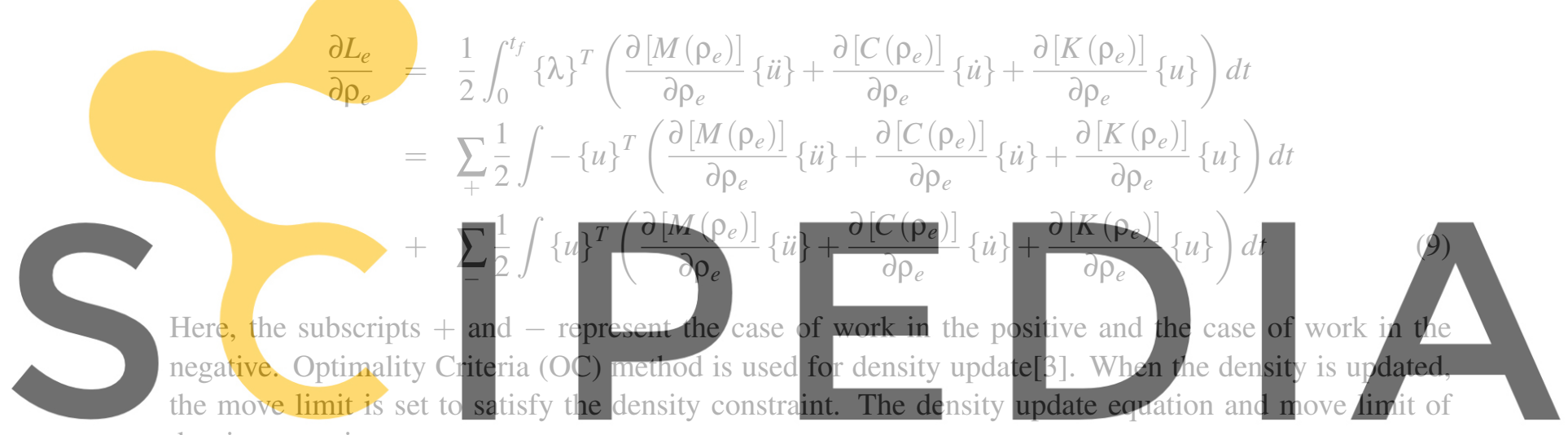

density are written as

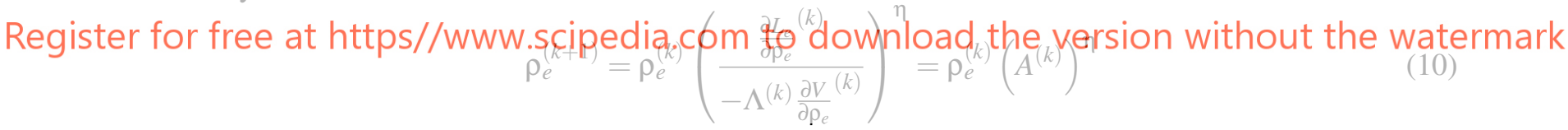

$$
\begin{aligned}
& \rho_{e}^{(k+1)}= \begin{cases}\rho_{e}^{L^{(k)}} & \left(\rho_{e}^{(k)}\left(A^{(k)}\right)^{\eta} \leq \rho_{e}^{L^{(k)}}\right) \\
\rho_{e}^{U^{(k)}} & \left(\rho_{e}^{(k)}\left(A^{(k)}\right)^{\eta} \geq \rho_{e}^{U^{(k)}}\right) \\
\rho_{e}^{(k)}\left(A^{(k)}\right)^{\eta} & (\text { otherwise })\end{cases} \\
& \rho_{e}^{L^{(k)}}=\max \left\{\rho_{e}^{(k)}-\varsigma, 0\right\} \\
& \rho_{e}^{U^{(k)}}=\min \left\{\rho_{e}^{(k)}+\varsigma, 1\right\}
\end{aligned}
$$

where $\zeta$ is the move limit of density, and $\Lambda$ is Lagrange multiplier. The solution of Lagrange multiplier $\Lambda$ is found by the bisection method. The SIMP method is often applied to topology optimization based on the density method[4]. In this study, the mass density, damping coefficient and Young's modulus are, respectively, represented by the SIMP method as follows

$$
\gamma_{e}\left(\rho_{e}\right)=\left(\gamma_{0}-\gamma_{\min }\right) \rho_{e}^{p_{m}}+\gamma_{\min }
$$




$$
\begin{gathered}
\zeta_{e}\left(\rho_{e}\right)=\left(1-\zeta_{\text {min }}\right) \rho_{e}^{p_{c}}+\zeta_{\text {min }} \\
E_{e}\left(\rho_{e}\right)=\left(E_{0}-E_{\text {min }}\right) \rho_{e}^{p_{k}}+E_{\text {min }}
\end{gathered}
$$

where $\gamma_{0}, E_{0}, \gamma_{\min }, \zeta_{\min }, E_{\min }, p_{m}, p_{c}$ and $p_{k}$ are respectively, the mass density of a material, the Young's modulus of a material, the mass density for numerical stability, the damping coefficient for numerical stability, the Young's modulus for numerical stability, the penalization parameter for mass density, the penalization parameter for damping coefficient and the penalization parameter for the Young's modulus. If the value of penalization parameters are changed, the values of sensitivity and physical property are also changed as shown in Figures 2 and 3.

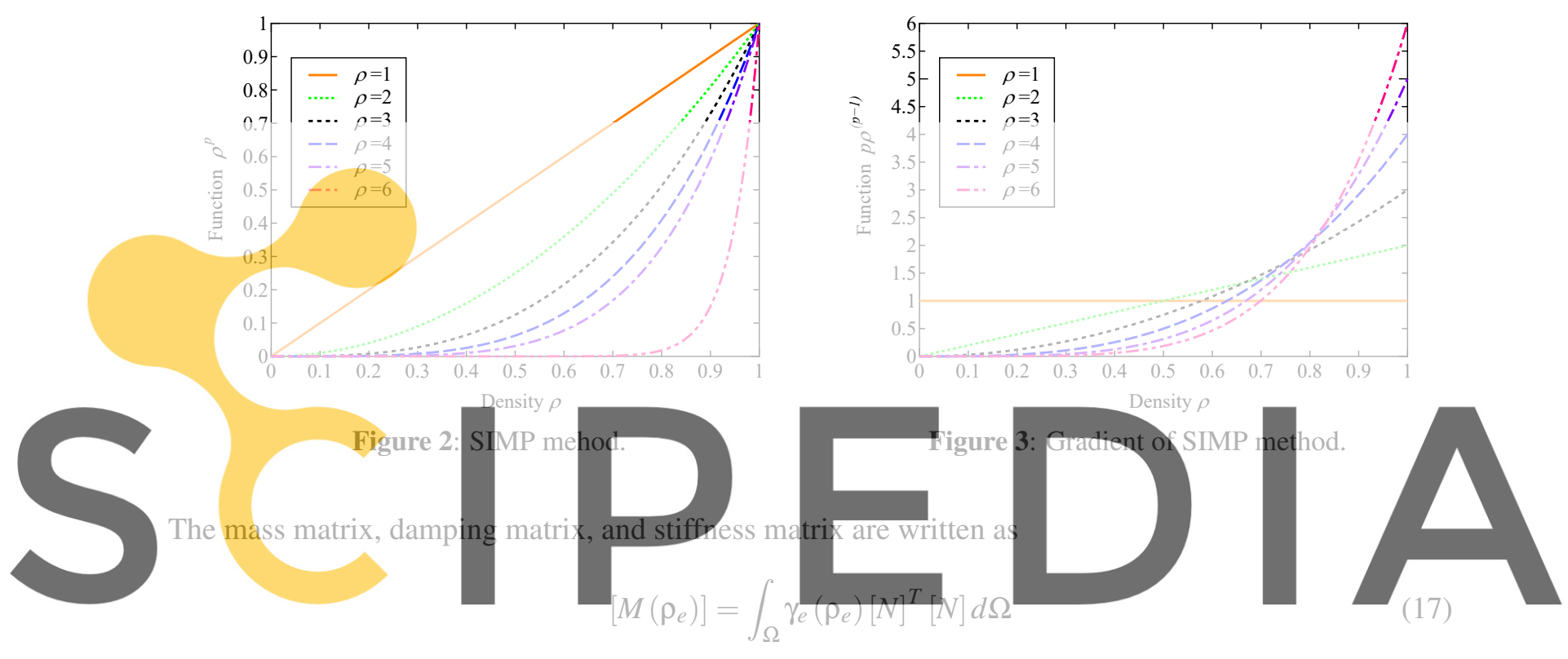

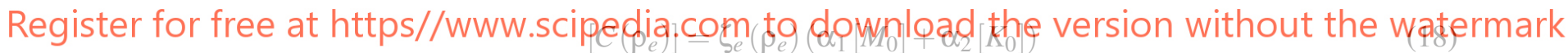

$$
\begin{gathered}
{\left[M_{0]}\right]=\int_{\Omega} \gamma_{0}\left(\rho_{e}\right)[N]^{T}[N] d \Omega} \\
{\left[K_{0}\right]=\left.\int_{\Omega}[B]^{T}\left[D\left(E_{e}\left(\rho_{e}\right)\right)\right][B] d \Omega\right|_{E_{e}=E_{0}}} \\
{\left[K\left(\rho_{e}\right)\right]=\int_{\Omega}[B]^{T}\left[D\left(E_{e}\left(\rho_{e}\right)\right)\right][B] d \Omega}
\end{gathered}
$$

where $[N],[B],[D]$ and $\alpha$ are, respectively, the shape function, B-matrix, D-matrix and Rayleigh damping.

\section{COMPUTATIONAL FLOW}

This chapter describes the topology optimization flowchart described in the precious chapter.

Step 1: Input of computational conditions and setting a computational model.

Step 2: 3D linear elastic analysis based on the finite element method is performed to obtain the displacement. 
Step 3: The performance function is calculated with the displacement.

Step 4: If the judgment of convergence equation $\left|J^{(k)}-J^{(k-1)}\right|<\varepsilon$ is satisfied, the computational is finalized. Otherwise, go to the next step.

Step 5: Sensitivity analysis is performed using the gradient of the Lagrange function with respect to density $\rho$.

Step 6: The density is updated by the OC method. Then go back to step 2.

By setting the maximum number of iterations $k_{\max }$, the computational is finalized even if the judgment of convergence equation is not satisfied.

\section{COMPUTATIONAL CONDITIONS}

This chapter describes the calculation model and calculation conditions. In this study, the calculation model is the cantilever beam model as shown in Figure 4, the red arrow indicates uniformly distribution load, and the $Y-Z$ surface is fixed in all directions. The load is assumed to be the impulse waveform as shown in Figure 5. The model size is $40[\mathrm{~mm}] \times 10[\mathrm{~mm}] \times 32[\mathrm{~mm}]$, and an element is a cube with a side $1[\mathrm{~mm}]$. Other common conditions are as shown in Table 1. As mentioned in Chapter 1, in the unsteady oscillation problem for the cantilever beam model, the optimal density distribution is changed by the scale of each sensitivity term. And, in the topology optimization based on the SIMP method, the physical property and sensitivity are defined by penalization parameters. Thus, in this study, comparative studies are conducted when one penalization parameter is changed, as shown in Table 2.
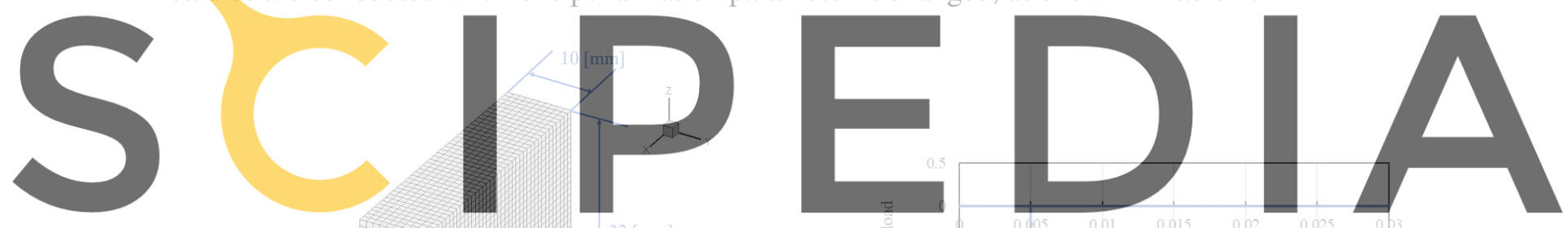

Register for free at https//www.scipedia.cing to dowhload the version without the watermark distributed load

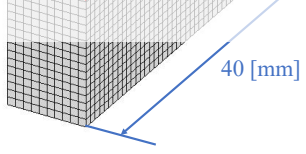

Figure 4: Cantilever model with boundary conditions.
Figure 5: Relationship between load and time. 
Table 1: Computational conditions.

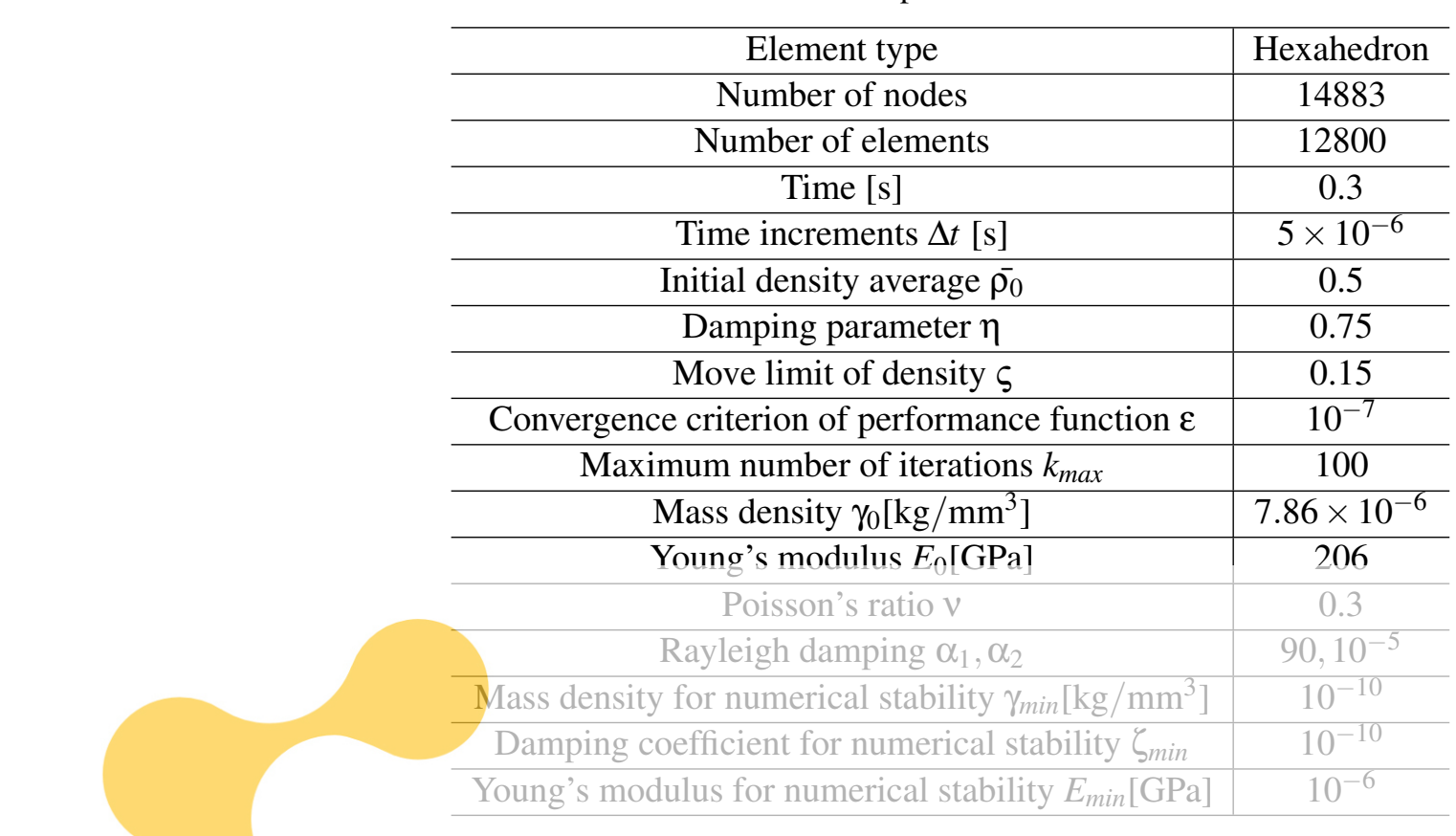
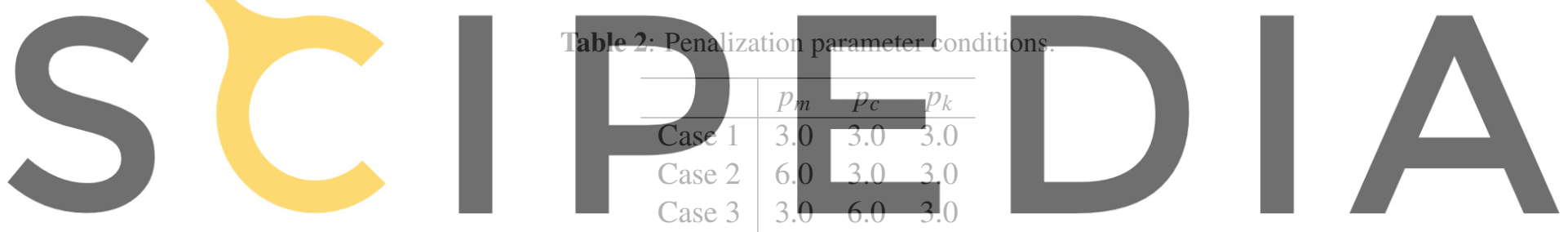

\section{Register for free at https//www.scipedia.com to download the version without the watermark}

\section{RESULTS OF NUMERICAL ANALYSIS}

In this chapter, the results of topology optimization analysis in unsteady oscillation problem for the cantilever beam model are described. Figures 6 to 9 show the results of density distribution. Here, the elements with a density of 0.2 or less are eliminated. The left view of these figures represents an isometric view, and the right view represents a side view. In addition, Figure 10 shows the displacement waveform in $Z$ direction at the load point, and Figure 11 and Table 3 show the performance function history and the value of performance function at the convergence, respectively. First, from the results of the density distribution, different density distributions were obtained by changing the penalization parameters, $p_{m}, p_{c}$ and $p_{k}$. However, there is no big difference. The density distributions of Cases 1 and 2 are like a solid. In contrast, the density distributions of Cases 3 and 4 are like a hollow. Comparing the side views of Cases 1 and 2, it can be seen that the side view of Case 2 has a lot of density distribution at the tip. The result of Case 4 has the most density distribution at fixed end of all Cases. Next, the results of the displacement waveform and performance function are described. If the penalization parameters are changed, the displacement waveform and performance function are smaller than the result of Case 
1. In particular, the result of Case 4 has the smallest displacement waveform and performance function. On the other hand, the performance functions of Cases 1 and 3 have almost the same value. With these results in mind, we found two points. The first point is that the results of the smallest displacement waveform and performance function can be obtained by increased the penalization parameter $p_{k}$. The second point is that the displacement waveform and performance function do not improve even if the penalization parameter $p_{c}$ is increased.

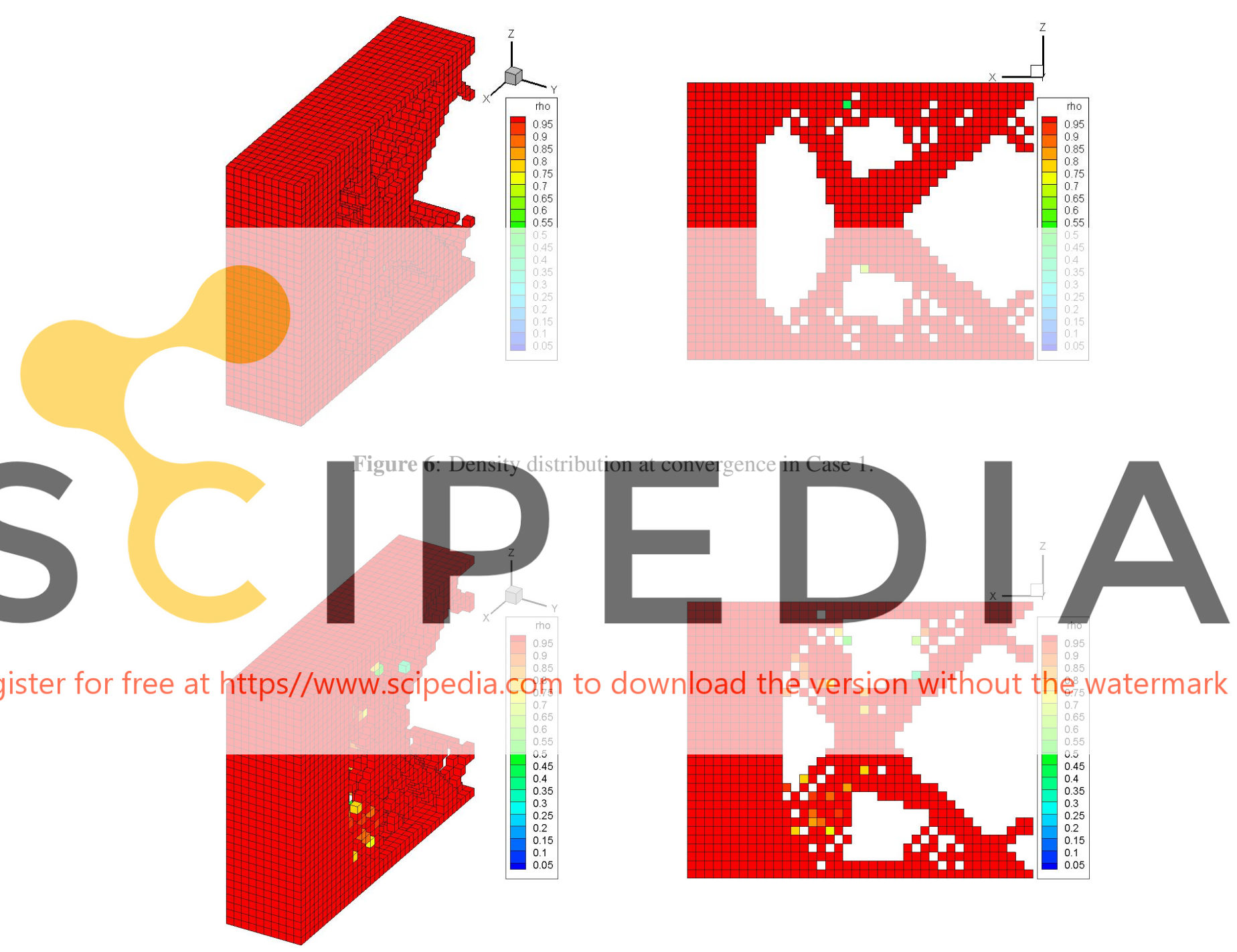

Figure 7: Density distribution at convergence in Case 2. 

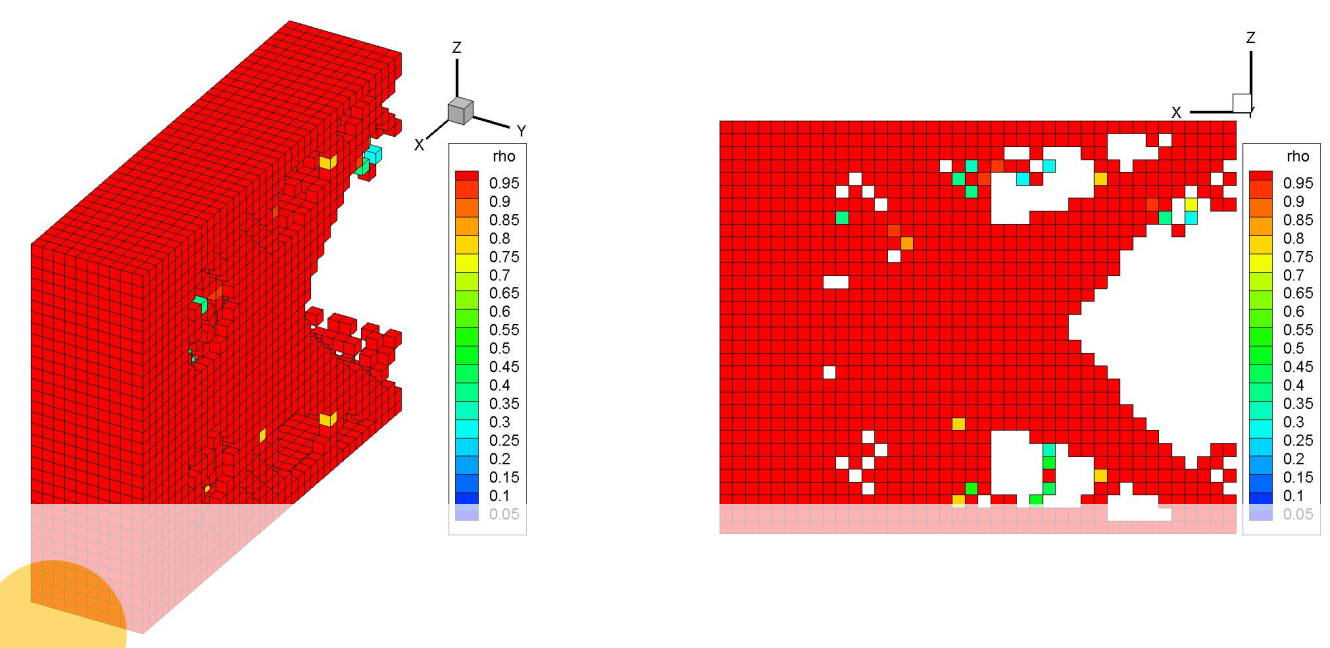

Figure 8: Density distribution at convergence in Case 3.
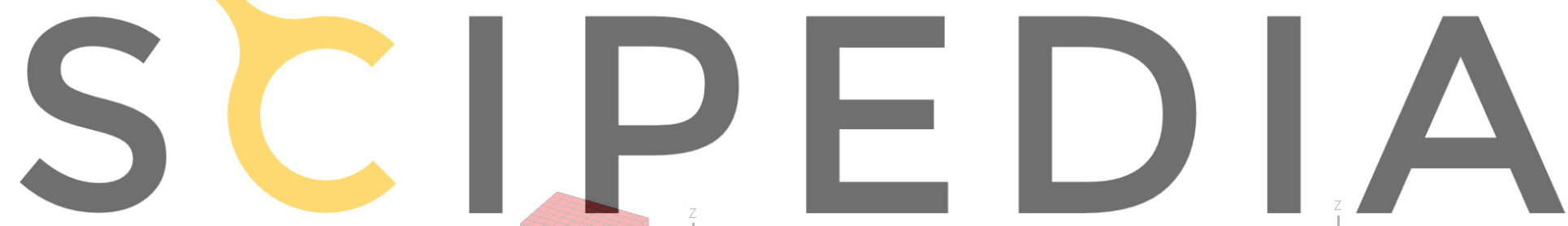

Register for free at https//www.scipedia.com to download the version without the watermark
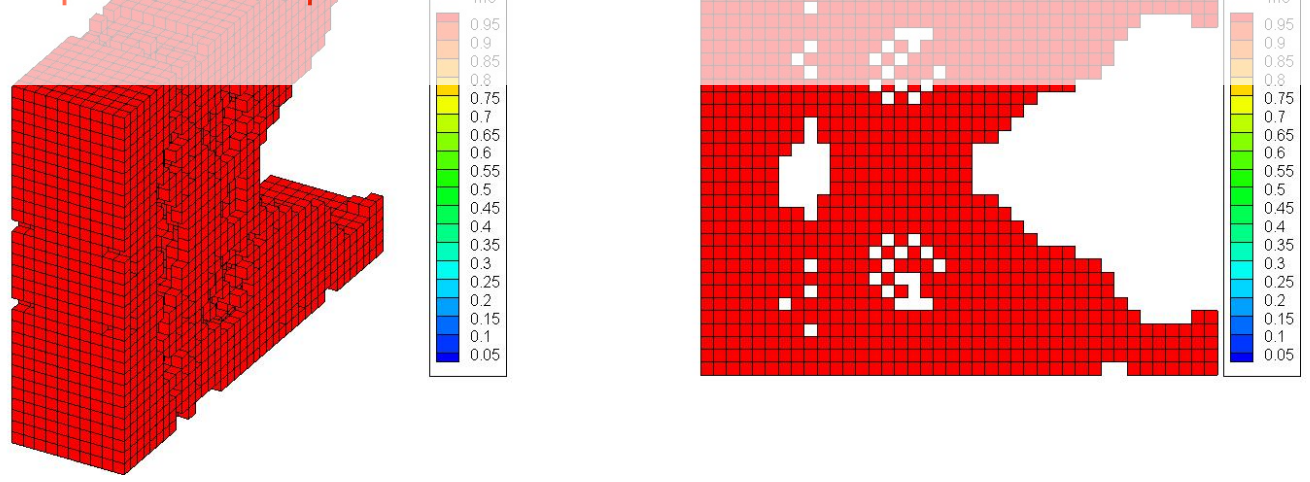

Figure 9: Density distribution at convergence in Case 4. 


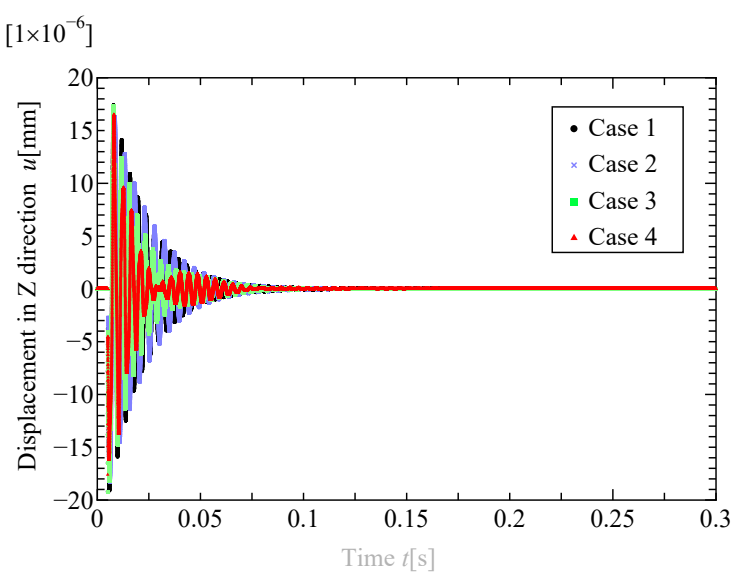

(a)All times.

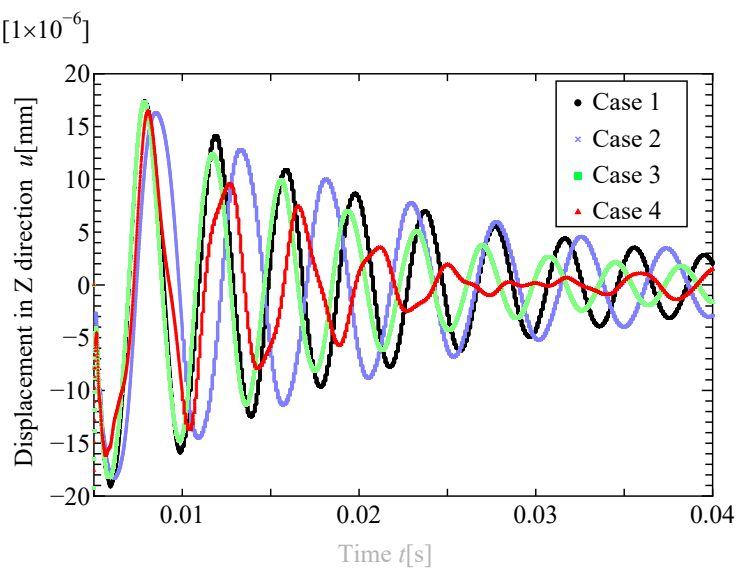

(b)Detail.

Figure 10: Displacement waveform in $Z$ direction.
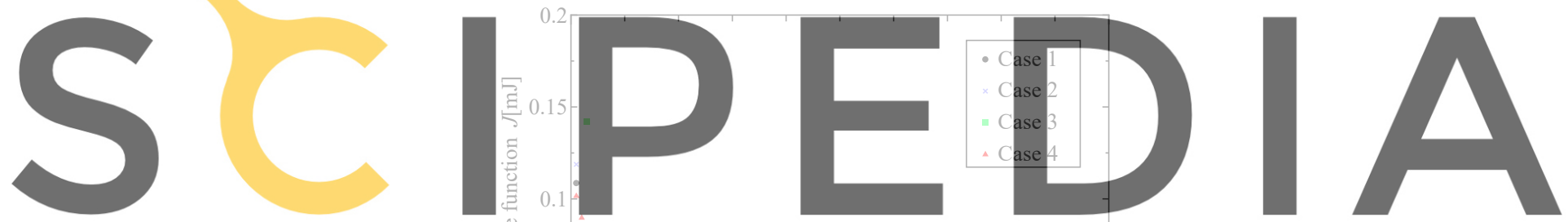

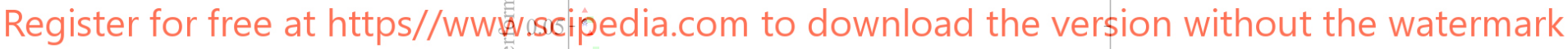

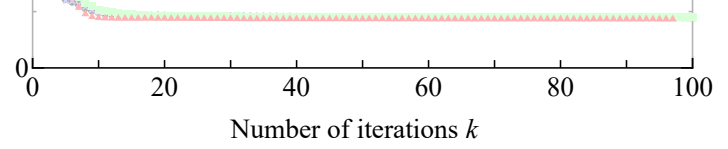

Figure 11: Performance function history.

Table 3: Penalization parameter conditions and converged performance function.

\begin{tabular}{l|cccc}
\hline & $p_{m}$ & $p_{c}$ & $p_{k}$ & Performance function $J[\mathrm{~J}]$ \\
\hline Case 1 & 3.0 & 3.0 & 3.0 & $2.25 \times 10^{-5}$ \\
Case 2 & 6.0 & 3.0 & 3.0 & $2.19 \times 10^{-5}$ \\
Case 3 & 3.0 & 6.0 & 3.0 & $2.24 \times 10^{-5}$ \\
Case 4 & 3.0 & 3.0 & 6.0 & $2.16 \times 10^{-5}$ \\
\hline
\end{tabular}




\section{CONCLUSION}

This paper is written the unsteady oscillation problem in topology optimization for a cantilever beam model. The first study was divided the optimization problem into maximization problem and minimization problem by classified the strain energy into the work in the positive and the work in the negative. Thereby, the self-adjoint relationships were derived. The second study was investigated the relationship between each sensitivity term and the result of topology optimization. Sensitivity was classified into three terms, and the ratio of each sensitivity was changed by changing the penalization parameters of the SIMP method. As a result, the displacement waveform and performance function were the smallest when the penalization parameter $p_{k}$ was increased. In contrast, when the penalization parameter $p_{c}$ was increased, the results were not much different from when the penalization parameters were the same. Therefore, it was found that the sensitivity of the stiffness matrix $[K]$ is the most important when the strain energy is used as the performance function.

\section{ACKNOWLEDGMENTS}

This work was supported by Grant-in-Aid for Scientific Research (C) Grant No. 18K03897 and funds for joint research from Oiles Corporation. Most of the topology optimization was carried out using the supercomputer system at Kyushu University's Institute for Information Technology. We wish to thank all the persons who assisted us in this study.

\section{REFERENCES}

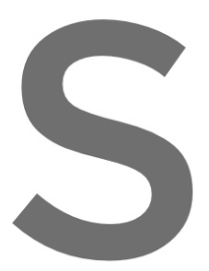

[1] Bendsøe M. P. and Kikuchi N., Generaxing opt
enization method, Comput. Methods in Appl.
[2] Bendsøe M. P. and Sigmund O., Topology
Springer-Verlag Berlin Heidelberg GmbH, (200
[3] Suzuki K. and Kikuchi N., A homogenization
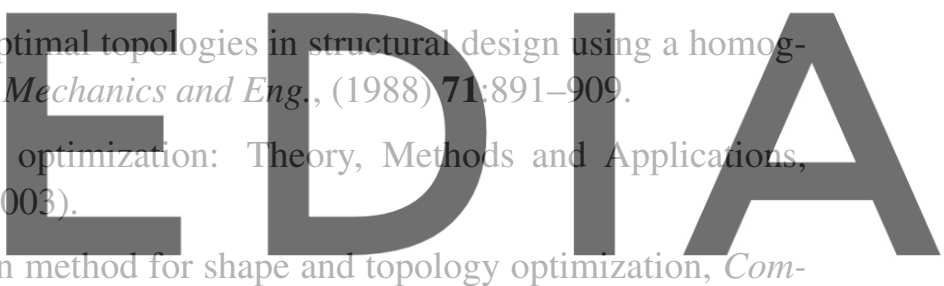

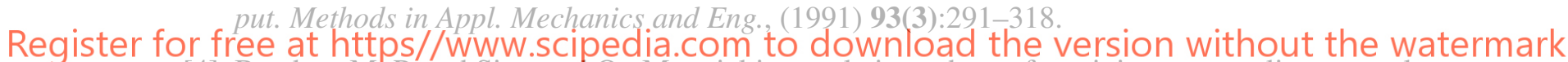

[4] Bendsøe M. P. and Sigmund O., Material interpolation scheme for minimum compliance topology

optimization, Archive of Appl. Mechanics, (1999) 69(9):635-654. 